1 A randomized controlled trial of the effects of $\mathbf{n}-\mathbf{3}$ fatty acids on resolvins in

$9 \quad{ }^{1}$ School of Medicine and Pharmacology, Royal Perth Hospital Unit, University of Western

\section{chronic kidney disease}

Emilie Mas ${ }^{1}$, Anne Barden ${ }^{1} *$, Valerie Burke ${ }^{1}$, Lawrence J. Beilin ${ }^{1}$, Gerald F Watts ${ }^{1}$, Rae-Chi Huang ${ }^{12}$, Ian B. Puddey ${ }^{1}$, Ashley B. Irish ${ }^{3}$, Trevor A Mori ${ }^{1}$

* Equal first authorship

Australia, ${ }^{2}$ Telethon Kid’s Institute, and ${ }^{3}$ Department of Nephrology and Transplantation, Royal Perth Hospital, Perth, WA 6000, Australia.

Address for correspondence: Dr Emilie Mas, School of Medicine and Pharmacology, University of Western Australia, Medical Research Foundation Building, GPO BOX X2213, PERTH Western Australia 6847. Phone: 6189224 0259, Fax: 6189224 0246, Email: emilie.mas@uwa.edu.au

Keywords: resolvins, fish oil, inflammation resolution, renal disease

Short title: $\mathrm{n}-3$ fatty acids and resolution of inflammation in renal disease

Abbreviations: eicosapentaenoic acid (EPA), docosahexaenoic acid (DHA), liquid chromatography-tandem mass spectrometry (LC-MS/MS), 18R/S-hydroxy-5Z, 8Z, 11Z, 14Z, 16E-eicosapentaenoic acid (18-HEPE), 17S-hydroxy-4Z, 7Z, 10Z, 13Z, 15E, 19Zdocosahexaenoic acid (17-HDHA), 7S,8R,17S-trihydroxy-4Z,9E,11E,13Z,15E,19Zdocosahexaenoic acid (RvD1), 7S,8R,17R-trihydroxy-4Z,9E,11E,13Z,15E19Z- 
26 docosahexaenoic acid (17R-RvD1), 7S,16R,17S-trihydroxy-4Z,8E,10Z,12E,14E,19Z-

27 docosahexaenoic acid (RvD2), 10S,17S-dihydroxy-4Z,7Z,11E,13Z,15E,19Z-

28 docosahexaenoic acid (10S,17S-diHDHA), 10R,17S-dihydroxy-4Z,7Z,11E,13E,15Z,19Z-

29 docosahexaenoic acid (protectin D1, PD1), leukotriene $\mathrm{B}_{4}-\mathrm{d}_{4} \quad\left(\mathrm{LTB}_{4}-\mathrm{d}_{4}\right)$,

30 ethylenediaminetetraacetic acid (EDTA); cardiovascular disease (CVD); chronic kidney

31 disease (CKD); specialized proresolving lipid mediator (SPM); coenzyme Q10 (CoQ); end

32 stage renal disease (ESRD); cyclooxygenase-2 (COX-2); butylated hydroxytoluene (BHT);

33 reduced glutathione (GSH); leukotriene B4-deuterated $\left(\mathrm{LTB}_{4}\right.$-d4); tumor necrosis factor-

34 alpha (TNF- $\alpha$ ); interleukin-10 (IL-10); resolvin E1 (RvE1); body mass index (BMI);

35 estimated glomerular filtration rate (eGFR). 


\section{Abstract}

Background and objective: The high incidence of cardiovascular disease (CVD) in chronic kidney disease (CKD) is related partially to chronic inflammation. n-3 Fatty acids have been shown to have anti-inflammatory effects and to reduce the risk of CVD. Specialized Proresolving Lipid Mediators (SPMs) derived from the n-3 fatty acids, eicosapentaenoic acid (EPA) and docosahexaenoic acid (DHA) actively promote the resolution of inflammation. This study evaluates the effects of n-3 fatty acid supplementation on plasma SPMs in patients with CKD.

Methods: In a double-blind, placebo-controlled intervention of factorial design, 85 patients were randomized to either n-3 fatty acids (4 g), Coenzyme $\mathrm{Q}_{10}$ (CoQ) (200 mg), both supplements, or control (4 g olive oil), daily for 8 weeks. The SPMs 18-HEPE, 17-HDHA, RvD1, 17R-RvD1, and RvD2, were measured in plasma by liquid chromatography-tandem mass spectrometry before and after intervention.

Results: Seventy four patients completed the 8 weeks intervention. n-3 Fatty acids but not CoQ significantly increased $(P<0.0001)$ plasma levels of 18 -HEPE and 17-HDHA, the upstream precursors to the E- and D- series resolvins, respectively. RvD1 was significantly increased $(P=0.036)$ after n-3 fatty acids, but no change was seen in other SPMs. In regression analysis the increase in 18-HEPE and 17-HDHA after n-3 fatty acids was significantly predicted by the change in platelet EPA and DHA, respectively.

Conclusion: SPMs are increased after 8 weeks n-3 fatty acid supplementation in patients with CKD. This may have important implications for limiting ongoing low grade inflammation in CKD. 


\section{Introduction}

Individuals with chronic kidney disease (CKD) have up to a 10-20 fold greater risk of cardiac death than age and sex-matched controls ${ }^{1}$. CKD is associated with significant patient morbidity and mortality and the treatment of CKD by dialysis makes a large contribution to the growing health care costs. More than $50 \%$ of deaths in stage 5 CKD patients receiving maintenance dialysis are due to cardiovascular disease (CVD), and the risk of coronary artery disease increases exponentially with declining kidney function ${ }^{2,3}$. In the National Health and Nutrition Examination Survey (NHANES II), renal function of less than $70 \mathrm{ml} / \mathrm{min} / 1.73 \mathrm{~m}^{2}$ associated with a 51\% increase in CVD death risk ${ }^{4}$, while the Atherosclerosis Risk in Communities Study ${ }^{5}$ showed that GFR $>15$ and $<59 \mathrm{ml} / \mathrm{min} / 1.73 \mathrm{~m}^{2}$ associated with a $38 \%$ increase in risk of CVD death The increased incidence of CVD in CKD is explained in part, by an increased prevalence of traditional risk factors such as hypertension, diabetes mellitus, dyslipidemia, smoking, obesity and physical inactivity, and non-traditional risk factors including anaemia, abnormal calcium/phosphate metabolism, inflammation, malnutrition, oxidative stress, and elevated lipoprotein (a) ${ }^{1}$. CKD is now considered a risk factor for allcause mortality independent of CVD risk ${ }^{2,3,6,7}$.

Inflammation plays an important role in acute and chronic kidney injury and may contribute to glomerular and tubulointerstitial damage. Unresolved inflammation promotes progressive glomerulosclerosis and interstitial fibrosis manifest as proteinuria and eventual renal failure ${ }^{8,9}$. Resolution of inflammation is an active process regulated by novel autacoids known as Specialized Proresolving Lipid Mediators (SPMs) ${ }^{10,11}$. SPMs are generated locally by polymorphonuclear leukocytes during the resolution of inflammation and include lipoxins derived from the n-6 fatty acid arachidonic acid (AA, 20:4n-6), and resolvins, protectins and maresins derived from the n-3 fatty acids eicosapentaenoic acid (EPA, 20:5n-3) and docosahexaenoic acid (DHA, 22:6n-3) ${ }^{10}$. Several families of chemically and functionally 
distinct SPMs have been identified including E-series resolvins derived from EPA via P450 metabolism or aspirin-acetylated cyclooxygenase (COX-2), and D-series resolvins, protectins/neuroprotectins and maresins derived from DHA via lipoxygenase or aspirin acetylated COX-2 ${ }^{11}$. SPMs act at picogram-nanogram concentrations in vivo and directly block and limit excessive polymorphonuclear leukocyte chemotaxis. They inhibit proinflammatory cytokine production, increase anti-inflammatory cytokine synthesis, and activate specific G-coupled protein receptors on neutrophils and macrophages to enhance clearance of cellular debris that is required for tissue homeostasis to be re-established ${ }^{11,12}$.

n-3 fatty acids have been associated with cardiovascular protection and improve cardiovascular disease risk factors such as blood pressure, plasma triglycerides and inflammation ${ }^{13,14}$. We have also shown that $n-3$ fatty acid supplementation results in elevated levels of SPMs in healthy volunteers ${ }^{15,16}$ suggesting that they may contribute to altered immune function. In a randomized controlled trial that examined the main and additive effects of n-3 fatty acids and coenzyme Q10 (CoQ) on cardiovascular risk in patients with CKD we showed that n-3 fatty acid supplementation reduced blood pressure, heart rate and plasma triglycerides ${ }^{17}$. As there is no evidence to suggest that CoQ affects SPM, this study utilized plasma samples from that trial ${ }^{17}$ to assess how n-3 fatty acid supplementation affected plasma SPM using a main effects analysis.

\section{Materials and Methods}

\subsection{Study population}

Men and women with chronic renal impairment, aged 25-75 years, were recruited from the renal units of Royal Perth, Sir Charles Gairdner and Fremantle Hospitals, in Perth, Western Australia. All participants had estimated (e)GFR $>15$ and $<60 \mathrm{ml} / \mathrm{min} / 1.73 \mathrm{~m}^{2}$, and 
110 serum creatinine $<350 \mathrm{mmol} / \mathrm{l}^{18}$. Patients were current nonsmokers and were excluded if 111 they had angina pectoris; major surgery; a cardiovascular event or diagnosis of CVD; BP >

112 170/100mmHg; diabetes; liver disease; nephrotic syndrome (proteinuria >3 g/day or 113 protein/creatinine ratio $>300 \mathrm{mg} / \mathrm{mmol}$ ); or haemoglobin $<110 \mathrm{~g} / \mathrm{l}$. Patients were excluded if 114 they regularly took nonsteroidal anti-inflammatory or immunosuppressive drugs, nitrates 115 (including Viagra); ate $\geq 1$ fish meal per week or regularly took fish oil supplements; or if 116 they consumed an average of $>4$ standard alcoholic drinks/day. Antihypertensive or lipid117 lowering medication were not criteria for exclusion. The study was approved by the ethics committees of the three hospitals in accordance with the declaration of Helsinki and all patients gave informed written consent. The study was registered with the Australian Clinical Trials Register (ACTRN012605000088640). The CONSORT statement for this trial has been published with the main outcomes from this trial ${ }^{17}$. 
control, and 2 X 50 mg CoQ or placebo, twice daily with meals.

Volunteers were asked to maintain their usual diets, medications, alcohol intake and physical activity and not to alter their lifestyle during the intervention. All measurements were performed at baseline and during the last week of intervention. Compliance with the supplements was monitored by capsule count and measurement of platelet fatty acids.

\subsection{Measurement of fatty acid composition}

Platelet phospholipid fatty acids are recognised as a reliable measure of compliance with fatty acids intake. This measure was used to determine the compliance with n-3 fatty acid intake in the patients. Platelet phospholipid fatty acids were measured by gas chromatography as previously described ${ }^{19}$. Samples were extracted with $2 \mathrm{ml}$ chloroform/methanol (2:1; vol:vol). Fatty acid methyl esters were analysed by gas liquid chromatography using an Agilent Technologies model 7890A gas chromatograph (Santa Clara, CA). The column was a Supelco SP-2560 (100 m x 0.25 mm ID x $0.20 \mu \mathrm{m}$; Bellefonte, PA) with a temperature program as follows: $180^{\circ} \mathrm{C}(1.75 \mathrm{~min})$, then $5^{\circ} \mathrm{C} / \mathrm{min}$ to $200^{\circ} \mathrm{C}$ (held $1.75 \mathrm{~min}$ ), then $10^{\circ} \mathrm{C} / \mathrm{min}$ to $240^{\circ} \mathrm{C}$ (held $4.5 \mathrm{~min}$ ) using hydrogen as carrier gas at a split ratio of 30:1. Peaks were identified by comparison with a known standard mixture.

\subsection{Measurement of SPMs}

Fasted blood samples were collected into EDTA/BHT/GSH for measurement of plasma SPMs. Baseline and end of intervention samples were measured in the same assay to minimize within-subject variation. Briefly, plasma $(1 \mathrm{ml})$ and internal standard leukotriene $\mathrm{B}_{4}-\mathrm{d}_{4}\left(\mathrm{LTB}_{4}-\mathrm{d}_{4}\right)(500 \mathrm{pg})$ were acidified with $2 \mathrm{ml}$ of $100 \mathrm{mM}$ sodium acetate $\mathrm{pH} 3$, applied to solid phase extraction cartridges (Bond Elut C18 500mg, Agilent Technologies, Lake Forrest, CA, USA), and washed with water and hexane. The SPMs were eluted with ethyl acetate 

and methanol (50/50; v/v) for analysis by LC-MS/MS (injection volume $50 \mu \mathrm{l}$ ). The standards 18R/S-hydroxy-5Z, 8Z, 11Z, 14Z, 16E-eicosapentaenoic acid (18-HEPE), 17Shydroxy-4E, 7Z, 10Z, 13Z, 15Z, 19Z-docosahexaenoic acid (17-HDHA), 7S,8R,17Rtrihydroxy-4Z,9E,11E,13Z,15E19Z-docosahexaenoic acid $\quad$ (17R-RvD1), 7S,16R,17Strihydroxy-4Z,8E,10Z,12E,14E,19Z-docosahexaenoic acid (RvD2), 10S,17S-dihydroxy4Z,7Z,11E,13Z,15E,19Z-docosahexaenoic acid (10S,17S-diHDHA) and leukotriene $\mathrm{B}_{4}$ - $\mathrm{d}_{4}$ $\left(\mathrm{LTB}_{4}-\mathrm{d}_{4}\right)$ were purchased from Cayman Chemicals (Ann Arbor, MI, USA). The PD1 standard was kindly provided as a gift by Professor Charles N. Serhan (Harvard Medical School, Boston, Massachusetts, USA). The SPMs were quantitated using a Thermo Scientific TSQ Quantum Ultra Triple Quadrupole LCMS System (ThermoFisher Scientific, Waltham, MA, USA) equipped with an electrospray ionization source (ESI) operated in negative ion mode. Liquid chromatography was performed on a Zorbax Eclipse XDB C18 column (2.1 x 100mm x 3.5um, Agilent Technologies, Santa Clara, CA, USA) as previously described by our group ${ }^{15,16}$.

\subsection{Statistical analysis}

Analyses included only participants who completed the trial. Baseline measures were compared using one-way analysis of variance. Univariate analysis was performed using SPSS 15.0 (SPSS Inc., Chicago, Illinois, USA) and assessed main effects and interactive effects of 8 weeks of n-3 fatty acids and CoQ treatment on plasma SPMs adjusting for baseline values. Regression analysis was used to assess the relationship between changes in platelet n-3 fatty acids, eGFR and C-reactive protein (CRP) with plasma SPMs at baseline and after intervention adjusting for baseline values. 


\section{Results}

186

187

\subsection{Patient Characteristics}

The CONSORT diagram for the study has been previously published, Mori et al. ${ }^{17}$. At baseline there were 63 men and 22 women aged $56.5 \pm 1.4$ years with a BMI of $27.3 \pm 0.5$ $\mathrm{kg} / \mathrm{m}^{2}$ and clinic BP of $125.0 \pm 1.7$ / 72.3 $\pm 0.9 \mathrm{mmHg}$. Mean eGFR was $35.8 \pm 1.2$ $\mathrm{ml} / \mathrm{min} / 1.73 \mathrm{~m}^{2}$ (range $17.3-58.1 \mathrm{ml} / \mathrm{min} / \mathrm{1}^{2} .73 \mathrm{~m}^{2}$ ) (stages $3-4 \mathrm{CKD}$ ) ${ }^{18}$. Baseline characteristics (Table 1) of the 74 patients that completed the trial (54 men and 20 women) confirmed the groups were well matched ${ }^{17}$.

\subsection{Effects of $\mathbf{n}-\mathbf{3}$ fatty acids on platelet phospholipid fatty acids}

Baseline platelet phospholipid fatty acids were not different between the groups (Table 2). The changes in long-chain n-3 fatty acids confirmed compliance with capsule intake (Table 2). EPA $(20: 5 n-3)$ and DHA $(22: 6 n-3)$ were both increased $(P<0.0001)$ and arachidonic acid $(20: 4 n-6)$ was reduced $(P=0.027)$ in the two groups consuming $n-3$ fatty acids relative to the groups not supplemented with n-3 fatty acids (Table 2).

\subsection{Effects of n-3 Fatty acids on Plasma SPMs}

Baseline concentrations of 18-HEPE, 17-HDHA, RvD2, RvD1 and 17R-RvD1 were not different between the groups (Table 3). There were no significant main effects of CoQ on plasma SPMs. The results are presented for the main effect of n-3 fatty acids after 8 weeks supplementation and compares the two groups taking n-3 fatty acids with the two groups not taking n-3 fatty acids. n-3 Fatty acids significantly increased plasma levels of the pathway precursors 18-HEPE (E-series resolvin from EPA) $(P<0.0001)$, and 17-HDHA (D-series resolvin from DHA) $(P<0.0001)$ (Table 3, Figure 1). Plasma RvD1 (D-series resolvin from 
DHA) was increased significantly $(P<0.05)$ after n-3 fatty acid supplementation (Table 3,

211

212

213

214 Figure 1), but no change was observed for the D-series resolvins 17R-RvD1 or RvD2 (Table 3). Plasma levels of 10S,17S-diHDHA and protectin PD1 were below the limit of quantification as assessed by Mas et al. ${ }^{15}$.

In regression analysis adjusting for baseline measures, the post-intervention concentration of plasma 18-HEPE and 17-HDHA were significantly related to the increased in platelet levels of EPA $(P<0.01)$ and DHA $(P<0.02)$, respectively. The relationship between the change in RvD1 and the change in platelet DHA after supplementation with n-3 fatty acids did not reach statistical significance $(P=0.062)$.

There were no significant relationships between any of the SPMs and renal function or CRP at baseline or after n-3 fatty acid supplementation.

\section{Discussion}

Our study has shown for the first time that supplementing patients with CKD for 8 weeks with $4 \mathrm{~g} / \mathrm{d}$ of n-3 fatty acids enhances the synthesis of SPMs that promote resolution of inflammation. This finding may have important implications related to limiting ongoing low grade inflammation in CKD. The study showed that n-3 fatty acids significantly increased RvD1 and the upstream precursors of the E-series and D-series resolvins, 18-HEPE and 17HDHA, respectively. RvD2 and 17R-RvD1 were not significantly different after n-3 fatty acid supplementation.

Plasma 18-HEPE in patients taking n-3 fatty acids was increased 4-5-fold relative to the group not taking n-3 fatty acids and was significantly related to the increase in platelet EPA. Plasma 17-HDHA was 1-2 fold higher in the n-3 fatty acid group and was significantly related to the increase in platelet DHA after n-3 fatty acid supplementation. Patients with CKD have reduced levels of plasma n-3 fatty acids compared with healthy individuals ${ }^{20}$, and 
thus a reduced capacity to synthesize SPMs under basal conditions. Therefore, the finding that supplementing CKD patients with n-3 fatty acids can reverse these deficiencies is clinically significant.

The fact that RvD1 and 17-HDHA were significantly increased with n-3 fatty acids in our study is important because both of these SPMs have been shown to be biologically active. In a mouse model of acute kidney injury, 17-HDHA and RvD1 are generated after ischemia reperfusion injury in plasma and kidney tissue with or without DHA administration suggesting they are important in renal injury ${ }^{21}$. The increase in 17-HDHA may be clinically important because it can affect a number of different immune mechanisms relevant to the progression of renal disease including promotion of phagocytosis ${ }^{22}$, suppression of the proinflammatory cytokines that mediate renal injury ${ }^{23}$, and activating differentiation of $\mathrm{B}$ cells into antibody secreting cells that are important for a functional humoral immune response $^{24}$.

In animal models, administration of RvD1 protects renal function and reduces morphologic renal injury if given before or within 10 minutes of inducing ischemia reperfusion injury ${ }^{21}$. RvD1 limits interstitial kidney fibrosis, reduces leukocyte accumulation and limits leukocyte activation ${ }^{21}$. These findings suggest that RvD1 can protect against the initial insult causing renal injury as well as limiting inflammation that associates with fibrosis and progression of renal disease. $\mathrm{RvD} 1$ is known to block macrophage Toll-like receptors a family of transmembrane proteins that mediate the inflammatory response ${ }^{11,21,25,26}$. This is relevant to renal injury as Toll-like receptors are present on kidney epithelial cells ${ }^{27}$ and have been implicated in the progression of the renal disease ${ }^{28}$. In animal models RvD1 has been shown to increase the synthesis of anti-inflammatory cytokines that protect against renal damage ${ }^{29,30}$. These effects may be partly due to inhibition of maladaptive activation of genes that cause leukocyte activation and adhesion ${ }^{31}$. 

a mouse model of renal fibrosis ${ }^{32}$. It is possible that like 18-HEPE, the E-series resolvins could have been elevated after n-3 fatty acid supplementation, however, we could not confirm this as the E-series resolvin standards were not available to us.

We have previously reported that $n-3$ fatty acid supplementation in these CKD patients did not affect renal function or CRP ${ }^{17}$. We found no relationship between SPMs at baseline or after supplementation with either renal function or CRP. There are mixed reports regarding the effects of n-3 fatty acids on CRP in CKD patients. CRP levels were unchanged in patients with stage 4-5 CKD, who were given $1.8 \mathrm{~g}$ or $3.6 \mathrm{~g}$ of $\mathrm{n}-3$ fatty acids daily for 10 weeks $^{33}$, and in hemodialysis patients that received $3 g /$ day of n-3 fatty acids for 2 months ${ }^{34}$. In contrast, a significant reduction in CRP was observed in hemodialysis patients after 4 months supplementation with n-3 fatty acids $(900 \mathrm{mg} / \mathrm{day})^{35}$, and in patients with end-stage renal disease who were given 1.56 g/day of $n-3$ fatty acids for 6 months ${ }^{36}$. It has been suggested that n-3 fatty acids are more effective when CRP is elevated at baseline and this may in part have contributed to the different study outcomes ${ }^{37,38}$.

Limitations in our study include the relatively short period of n-3 fatty acid supplementation, the small numbers of patients and the severity of renal disease. A larger study of longer duration in patients with more advanced renal disease may be necessary to see significant effects of n-3 fatty acids on renal function. We also did not measure other markers of inflammation such as cytokines in this study. Future studies measuring SPMs and cytokines may provide a broader insight into the mechanisms associated with any beneficial effect of n-3 fatty acids.

In conclusion, n-3 fatty acid supplementation for 8 weeks increases the synthesis of suggests that long term n-3 fatty acid supplementation is a potential therapy for limiting the 
low grade inflammation that associates with, and exacerbates, the progression of chronic kidney disease.

\section{Acknowledgements}

We thank Jackie Mansour, Christine Cowpland, Noeline Atkins and Lynette McCahon for diet counselling, nursing and technical assistance. We thank the renal physicians from Royal Perth Hospital (Dr Mark Thomas, Dr Barry Saker, Dr Kevin Warr), Fremantle Hospital (Dr Paolo Ferrari, Dr Helen Rhodes, Dr Hemant Kulkarni) and Sir Charles Gairdner Hospital (Dr Brian Hutchison, Dr Neil Boudville, Dr Grant Luxton, Dr Harry Moody, Dr Steven Richards) for their assistance in the recruitment of patients. Omacor ${ }^{\circledR}$ capsules were donated by Solvay Pharmaceuticals, Pymble, NSW, Australia. Coenzyme Q10 and placebo capsules were donated by Black mores Australia, Balgowlah, NSW, Australia. All the authors declared no competing interests.

\section{Statement of authorship}

Emilie Mas developed the LCMSMS method, analysed samples using mass spectrometry, assisted in the interpretation of the data and writing of the manuscript.

Valerie Burke performed the statistical analyses and contributed to the revision of the manuscript.

Anne Barden contributed to the statistical analysis, interpretation of results and writing of the manuscript.

Ashley B. Irish was involved in the study design, obtaining funding and recruitment of patients, interpretation of results and the revision of the manuscript.

Lawrence J. Beilin was involved in the study design, obtaining funding, interpretation of results and the revision of the manuscript. 
310 Gerald F Watts was involved in the study design, obtaining funding, interpretation of results

311 and the revision of the manuscript.

312 Ian B. Puddey was involved in the study design, obtaining funding, interpretation of results

313 and the revision of the manuscript.

314 Rae-Chi Huang was involved in obtaining funding, interpretation of results and the revision 315 of the manuscript.

316 Trevor A Mori is the principal investigator and was involved in the study design, obtaining

317 funding, interpretation of results and the revision of the manuscript.

318 All the authors have read the manuscript, agreed on the experimental findings, data 319 interpretation and presentation before submission. All authors read and approved the final 320 version of the paper.

321

322 Conflict of Interest statement

$323 \quad$ None

\section{Funding sources}

326 The study was supported by grants from the National Health and Medical Research Council 327 of Australia [APP303151 and APP1010495] and the National Heart Foundation of Australia 328 [G 09P 4280]. Emilie Mas is supported by the Medical Research Foundation of Royal Perth 329 Hospital. Trevor Mori and Rae-Chi Huang are National Health and Medical Research 330 Council of Australia Research Fellows. 
1. Johnson DW, Craven AM, Isbel NM. Modification of cardiovascular risk in hemodialysis patients: an evidence-based review. Hemodialysis international International Symposium on Home Hemodialysis. 2007;11:1-14.

2. Go AS, Chertow GM, Fan D, McCulloch CE, Hsu CY. Chronic kidney disease and the risks of death, cardiovascular events, and hospitalization. The New England journal of medicine. 2004;351:1296-305.

3. Briasoulis A, Bakris GL. Chronic kidney disease as a coronary artery disease risk equivalent. Current cardiology reports. 2013;15:340.

4. Muntner P, He J, Hamm L, Loria C, Whelton PK. Renal insufficiency and subsequent death resulting from cardiovascular disease in the United States. Journal of the American Society of Nephrology : JASN. 2002;13:745-53.

5. Manjunath G, Tighiouart H, Ibrahim H, MacLeod B, Salem DN, Griffith JL, et al. Level of kidney function as a risk factor for atherosclerotic cardiovascular outcomes in the community. Journal of the American College of Cardiology. 2003;41:47-55.

6. Baber U, Auguste U. Patients with chronic kidney disease/diabetes mellitus: the high-risk profile in acute coronary syndrome. Current cardiology reports. 2013;15:386. events in people with chronic kidney disease compared with those with diabetes: a population-level cohort study. Lancet. 2012;380:807-14.

8. Noronha IL, Fujihara CK, Zatz R. The inflammatory component in progressive renal disease--are interventions possible? Nephrology, dialysis, transplantation : official publication of the European Dialysis and Transplant Association - European Renal Association. 2002;17:363-8. 
9. Imig JD, Ryan MJ. Immune and inflammatory role in renal disease. Comprehensive Physiology. 2013;3:957-76.

10. Serhan CN, Chiang N. Resolution phase lipid mediators of inflammation: agonists of resolution. Curr Opin Pharmacol. 2013;13:632-40.

11. Serhan CN, Petasis NA. Resolvins and protectins in inflammation resolution. Chem Rev. 2011;111:5922-43.

12. Spite M, Claria J, Serhan CN. Resolvins, specialized proresolving lipid mediators, and their potential roles in metabolic diseases. Cell metabolism. 2014;19:21-36.

13. Breslow JL. n-3 fatty acids and cardiovascular disease. The American journal of clinical nutrition. 2006;83:1477S-82S.

14. Mori TA. Dietary n-3 PUFA and CVD: a review of the evidence. The Proceedings of the Nutrition Society. 2014;73:57-64.

15. Mas E, Croft KD, Zahra P, Barden A, Mori TA. Resolvins D1, D2, and other mediators of self-limited resolution of inflammation in human blood following n-3 fatty acid supplementation. Clinical chemistry. 2012;58:1476-84.

16. Barden A, Mas E, Croft KD, Phillips M, Mori TA. Short-Term n-3 Fatty Acid Supplementation but not Aspirin Increases Plasma Proresolving Mediators of Inflammation. J Lipid Res. 2014.

17. Mori TA, Burke V, Puddey I, Irish A, Cowpland CA, Beilin L, et al. The effects of omega-3 fatty acids and coenzyme Q10 on blood pressure and heart rate in chronic kidney

376 disease: a randomized controlled trial. Journal of hypertension. 2009;27:1863-72. 18. Foundation NK. K/DOQI clinical practice guidelines for chronic kidney disease: evaluation, classification, and stratification. American journal of kidney diseases : the official journal of the National Kidney Foundation. 2002;39:S1-266. 
19. Woodman RJ, Mori TA, Burke V, Puddey IB, Barden A, Watts GF, et al. Effects of purified eicosapentaenoic acid and docosahexaenoic acid on platelet, fibrinolytic and vascular function in hypertensive type 2 diabetic patients. Atherosclerosis. 2003;166:85-93.

20. Madsen T, Christensen JH, Svensson M, Witt PM, Toft E, Schmidt EB. Marine n-3 polyunsaturated fatty acids in patients with end-stage renal failure and in subjects without kidney disease: a comparative study. Journal of renal nutrition : the official journal of the Council on Renal Nutrition of the National Kidney Foundation. 2011;21:169-75.

21. Duffield JS, Hong S, Vaidya VS, Lu Y, Fredman G, Serhan CN, et al. Resolvin D series and protectin D1 mitigate acute kidney injury. J Immunol. 2006;177:5902-11.

22. Kohnke T, Gomolka B, Bilal S, Zhou X, Sun Y, Rothe M, et al. Acetylsalicylic Acid reduces the severity of dextran sodium sulfate-induced colitis and increases the formation of anti-inflammatory lipid mediators. BioMed research international. 2013;2013:748160.

23. Gonzalez-Periz A, Planaguma A, Gronert K, Miquel R, Lopez-Parra M, Titos E, et al. Docosahexaenoic acid (DHA) blunts liver injury by conversion to protective lipid mediators: protectin D1 and 17S-hydroxy-DHA. Faseb J. 2006;20:2537-9.

24. Ramon S, Gao F, Serhan CN, Phipps RP. Specialized proresolving mediators enhance human B cell differentiation to antibody-secreting cells. J Immunol. 2012;189:1036-42.

25. Hong S, Lu Y. Omega-3 fatty acid-derived resolvins and protectins in inflammation resolution and leukocyte functions: targeting novel lipid mediator pathways in mitigation of acute kidney injury. Frontiers in immunology. 2013;4:1-8.

26. Norling LV, Dalli J, Flower RJ, Serhan CN, Perretti M. Resolvin D1 limits polymorphonuclear leukocyte recruitment to inflammatory loci: receptor-dependent actions. Arterioscler Thromb Vasc Biol. 2012;32:1970-8.

27. Akcay A, Nguyen Q, Edelstein CL. Mediators of inflammation in acute kidney injury. Mediators of inflammation. 2009;2009:137072. 
28. Lu CY, Winterberg PD, Chen J, Hartono JR. Acute kidney injury: a conspiracy of Tolllike receptor 4 on endothelia, leukocytes, and tubules. Pediatr Nephrol. 2012;27:1847-54.

29. Krishnamoorthy S, Recchiuti A, Chiang N, Fredman G, Serhan CN. Resolvin D1 receptor stereoselectivity and regulation of inflammation and proresolving microRNAs. Am J Pathol. 2012;180:2018-27.

30. Krishnamoorthy S, Recchiuti A, Chiang N, Yacoubian S, Lee CH, Yang R, et al. Resolvin D1 binds human phagocytes with evidence for proresolving receptors. Proc Natl Acad Sci U S A. 2010;107:1660-5.

31. Deng J, Kohda Y, Chiao H, Wang Y, Hu X, Hewitt SM, et al. Interleukin-10 inhibits ischemic and cisplatin-induced acute renal injury. Kidney Int. 2001;60:2118-28.

32. Qu X, Zhang X, Yao J, Song J, Nikolic-Paterson DJ, Li J. Resolvins E1 and D1 inhibit interstitial fibrosis in the obstructed kidney via inhibition of local fibroblast proliferation. The Journal of pathology. 2012:506-19.

33. Guebre-Egziabher F, Debard C, Drai J, Denis L, Pesenti S, Bienvenu J, et al. Differential dose effect of fish oil on inflammation and adipose tissue gene expression in chronic kidney disease patients. Nutrition. 2013;29:730-6.

34. Tayyebi-Khosroshahi H, Houshyar J, Dehgan-Hesari R, Alikhah H, Vatankhah AM, Safaeian AR, et al. Effect of treatment with omega-3 fatty acids on C-reactive protein and tumor necrosis factor-alfa in hemodialysis patients. Saudi journal of kidney diseases and transplantation : an official publication of the Saudi Center for Organ Transplantation, Saudi Arabia. 2012;23:500-6.

35. Gharekhani A, Khatami MR, Dashti-Khavidaki S, Razeghi E, Noorbala AA, HashemiNazari SS, et al. The effect of omega-3 fatty acids on depressive symptoms and inflammatory markers in maintenance hemodialysis patients: a randomized, placebo-controlled clinical trial. European journal of clinical pharmacology. 2014;70:655-65. 
430 36. Bowden RG, Wilson RL, Deike E, Gentile M. Fish oil supplementation lowers C-reactive 431 protein levels independent of triglyceride reduction in patients with end-stage renal disease.

432 Nutrition in clinical practice : official publication of the American Society for Parenteral and 433 Enteral Nutrition. 2009;24:508-12.

434 37. Ciubotaru I, Lee YS, Wander RC. Dietary fish oil decreases C-reactive protein, 435 interleukin-6, and triacylglycerol to HDL-cholesterol ratio in postmenopausal women on 436 HRT. The Journal of nutritional biochemistry. 2003;14:513-21.

437 38. Zhao G, Etherton TD, Martin KR, Gillies PJ, West SG, Kris-Etherton PM. Dietary alpha438 linolenic acid inhibits proinflammatory cytokine production by peripheral blood mononuclear 439 cells in hypercholesterolemic subjects. The American journal of clinical nutrition. $440 \quad 2007 ; 85: 385-91$. 
444 Titles and legends

445 Table 1

446 Baseline characteristics of the groups

$447 \quad$ Table 2

448 Baseline and post-intervention platelet phospholipid fatty acids

449 Table 3

450 Plasma 18-HEPE, 17-HDHA, RvD1, 17R-RvD1 and RvD2 at baseline and post-intervention

451

452 Figure 1

453 Changes in plasma 18-HEPE, 17-HDHA and RvD1 after 8 weeks supplementation in the 2

454 groups not taking n-3 fatty acids (NO n-3 FA) compared with the groups taking n-3 fatty

455 acids (n-3 FA). Values are mean and SEM. General linear model analysis tested for the main

456 effects of n-3 fatty acids. Significance levels refer to post-intervention means adjusted for

457 baseline values * $P<0.05$, $+P<0.001$ for the effect of $n-3$ fatty acid supplementation

458

459

460 
Table 1: Baseline characteristics of the groups

\begin{tabular}{|c|c|c|c|c|}
\hline & Control & n-3 FA & CoQ & $\mathrm{n}-3 \mathrm{FA} \pm \mathrm{CoQ}$ \\
\hline $\mathbf{n}$ & 15 & 20 & 21 & 18 \\
\hline Men/Women (n/n) & $8 / 7$ & $12 / 8$ & $17 / 4$ & $17 / 1$ \\
\hline Age (years) & $58.6 \pm 2.6$ & $53.3 \pm 3.2$ & $55.4 \pm 2.7$ & $56.9 \pm 3.9$ \\
\hline BMI $\left(\mathrm{kg} / \mathrm{m}^{2}\right)$ & $27.6 \pm 1.7$ & $26.7 \pm 1.2$ & $26.6 \pm 0.9$ & $27.9 \pm 0.8$ \\
\hline Supine SBP (mmHg)* & $127.7 \pm 4.1$ & $126.3 \pm 3.4$ & $122.6 \pm 3.3$ & $124.5 \pm 4.6$ \\
\hline Supine DBP (mmHg)* & $71.9 \pm 2.6$ & $75.0 \pm 1.6$ & $72.9 \pm 1.6$ & $68.7 \pm 2.4$ \\
\hline eGFR $\left(\mathrm{ml} / \mathrm{min} / 1.73 \mathrm{~m}^{2}\right)$ & $34.6 \pm 2.3$ & $36.4 \pm 2.8$ & $38.8 \pm 2.2$ & $30.5 \pm 2.2$ \\
\hline Glucose (mmol/L) & $\begin{array}{c}4.9 \\
(4.6,5.2)\end{array}$ & $\begin{array}{c}4.8 \\
(4.4,5.1)\end{array}$ & $\begin{array}{c}4.7 \\
(4.3,5.1)\end{array}$ & $\begin{array}{c}5.0 \\
(4.7,5.3)\end{array}$ \\
\hline Insulin (mU/L) ${ }^{\S}$ & $\begin{array}{c}9.9 \\
(7.4,13.3) \\
\end{array}$ & $\begin{array}{c}10.4 \\
(7.8,13.8) \\
\end{array}$ & $\begin{array}{c}10.0 \\
(7.5,13.3)\end{array}$ & $\begin{array}{c}13.3 \\
(10.5,17.0) \\
\end{array}$ \\
\hline $\begin{array}{l}\text { C-Reactive Protein } \\
(\mathrm{mg} / \mathrm{L})^{\S}\end{array}$ & $\begin{array}{c}1.56 \\
(0.87,2.81) \\
\end{array}$ & $\begin{array}{c}1.74 \\
(0.99,3.06) \\
\end{array}$ & $\begin{array}{c}1.46 \\
(0.99,2.14)\end{array}$ & $\begin{array}{c}2.23 \\
(1.51,3.29) \\
\end{array}$ \\
\hline
\end{tabular}

*Average of 10 readings in the clinic using a Dinamap 1846 SX/P blood pressure monitor.

Values are Means \pm SEM or ${ }^{\S}$ Geometric mean (95\% confidence interval). 
Table 2. Baseline and post-intervention platelet phospholipid fatty acids

\begin{tabular}{|c|c|c|c|c|c|c|}
\hline \multirow[t]{2}{*}{$\%$ of Total Fatty acids } & \multirow{2}{*}{$\begin{array}{c}\text { Control } \\
(n=15)\end{array}$} & \multirow{2}{*}{$\begin{array}{c}n-3 \text { FA } \\
(n=20)\end{array}$} & \multirow{2}{*}{$\begin{array}{c}\mathrm{CoQ} \\
(\mathrm{n}=21)\end{array}$} & \multirow{2}{*}{$\begin{array}{c}\mathrm{n}-3 F A+ \\
\text { CoQ } \\
(n=18)\end{array}$} & \multicolumn{2}{|c|}{ Main Effects ( $P$ value) } \\
\hline & & & & & n-3FA & CoQ \\
\hline \multicolumn{7}{|l|}{ Platelet 20:4n6 (\%) } \\
\hline Baseline & $22.8 \pm 2.3$ & $25.4 \pm 1.6$ & $24.5 \pm 1.2$ & $24.9 \pm 1.6$ & & \\
\hline Post-Intervention & $23.6 \pm 1.9$ & $21.3 \pm 1.4$ & $24.7 \pm 1.2$ & $20.8 \pm 1.8$ & $-3.4 \pm 1.5 P=0.027$ & $0.07 \pm 1.5 P=0.963$ \\
\hline \multicolumn{7}{|l|}{ Platelet 20:5n3 (\%) } \\
\hline Baseline & $0.65 \pm 0.11$ & $0.69 \pm 0.09$ & $0.76 \pm 0.20$ & $0.61 \pm 0.09$ & & \\
\hline Post-Intervention & $0.57 \pm 0.05$ & $2.72 \pm 0.23$ & $0.61 \pm 0.06$ & $2.18 \pm 0.23$ & $1.87 \pm 0.17 P<0.0001$ & $-0.25 \pm 0.17 P=0.154$ \\
\hline \multicolumn{7}{|l|}{ Platelet 22:6n3 (\%) } \\
\hline Baseline & $2.06 \pm 0.24$ & $2.05 \pm 0.21$ & $2.22 \pm 0.12$ & $2.08 \pm 0.14$ & & \\
\hline Post-Intervention & $2.06 \pm 0.17$ & $3.00 \pm 0.27$ & $2.25 \pm 0.07$ & $3.06 \pm 0.26$ & $0.9 \pm 0.20 P<0.0001$ & $0.09 \pm 0.2 P=0.643$ \\
\hline
\end{tabular}

Values expressed as mean \pm SEM. n-3FA, n-3 fatty acid; ANOVA, analysis of variance; CoQ, coenzyme Q10; Baseline measures were compared by one-way ANOVA and were not significantly different between groups. General linear model analysis tested for main effects and interactions on post-intervention values adjusted for baseline value 
Table 3. Plasma 18-HEPE, 17-HDHA, RvD1, 17R-RvD1 and RvD2 at baseline and post-intervention

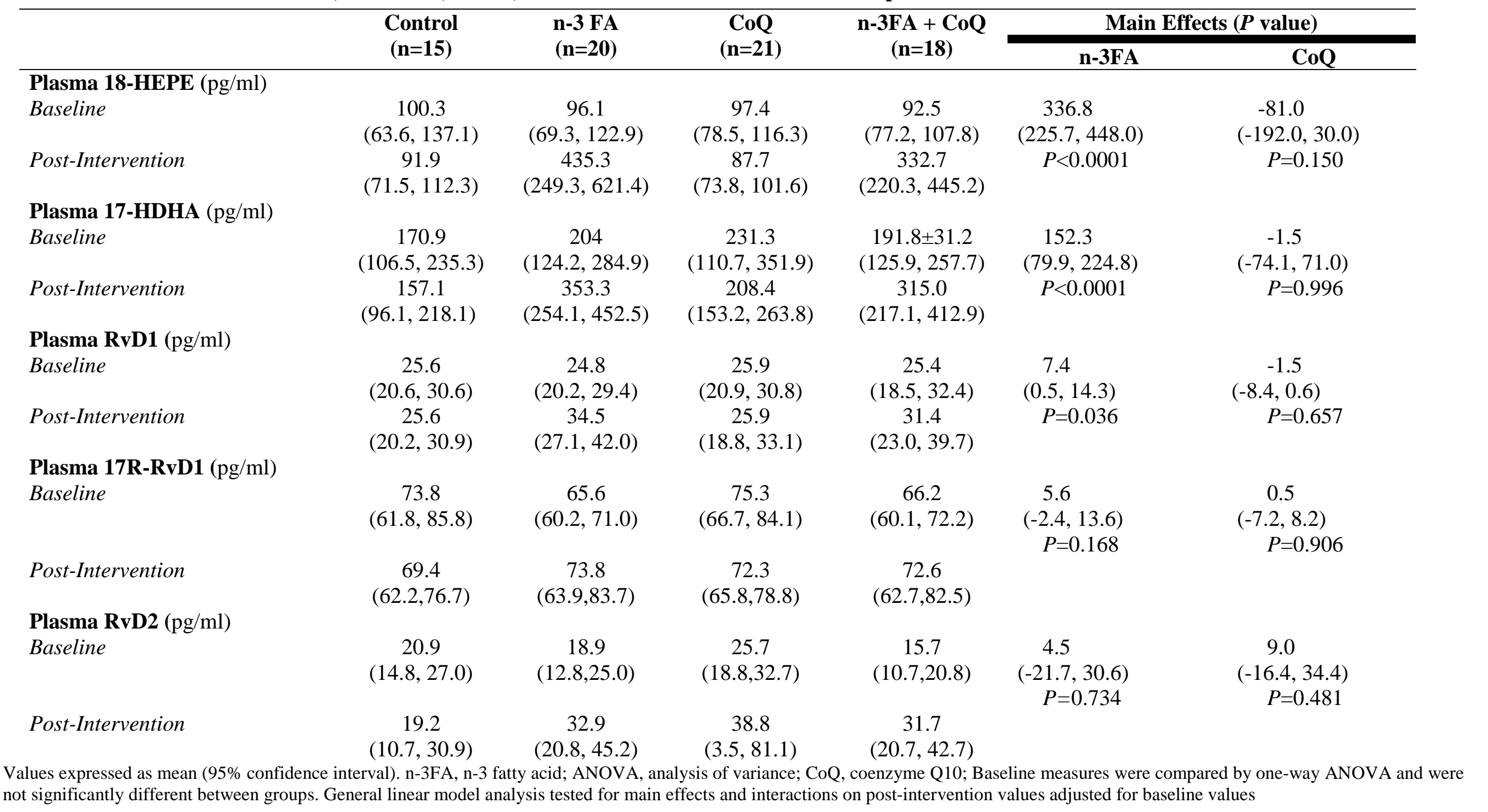


$1 \quad$ Figure 1

\section{8-HEPE}

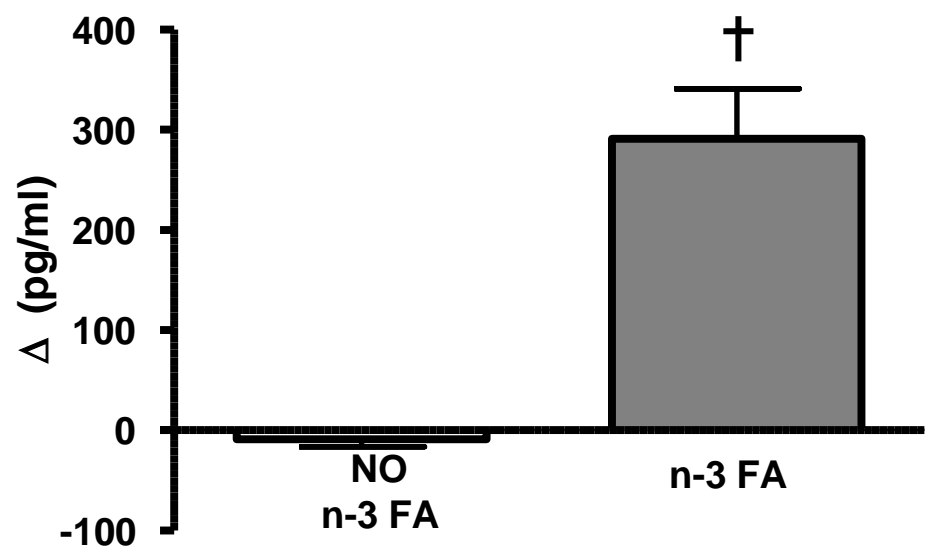

17-HDHA
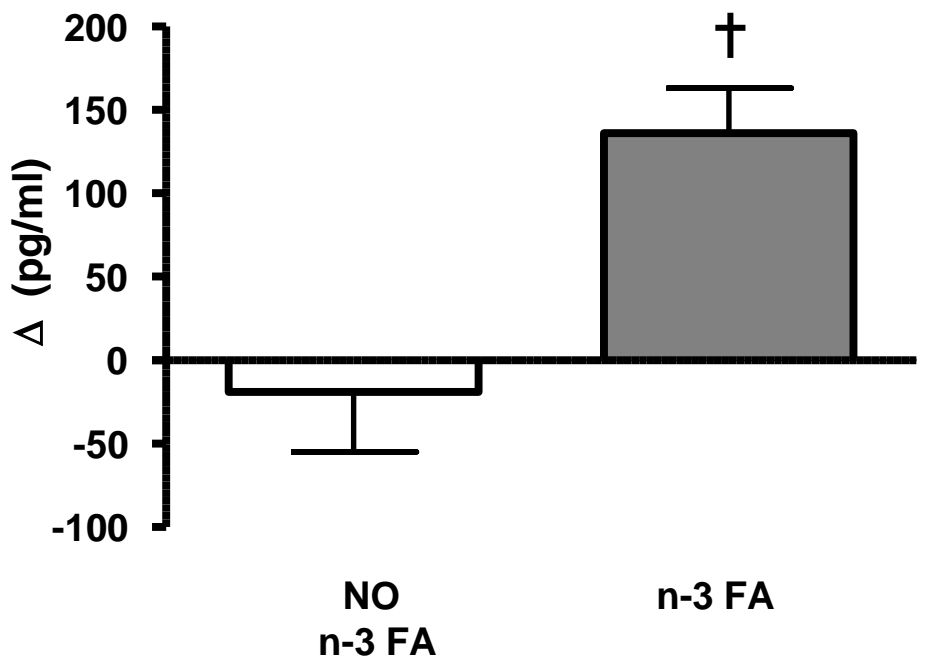

RvD1

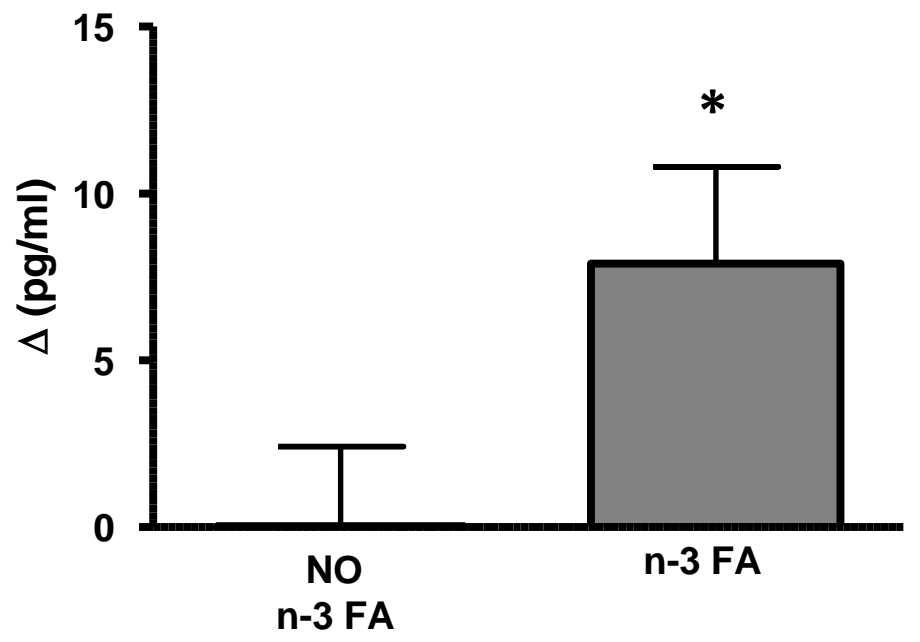

